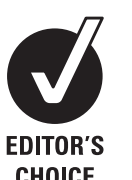

CHOICE

\section{See Editorial, p 734}

${ }^{1}$ Division of Cardiology, Department of Cardiological, Thoracic and Vascular Sciences, University of Padua, Padua, Italy ${ }^{2}$ Department of Internal Medicine, Ospedali Riuniti, Bergamo, Italy ${ }^{3}$ Department of Rheumatology, University of Padua, Padua, Italy ${ }^{4}$ Department of Emergency Medicine, Niguarda Hospital, Milan, Italy

${ }^{5}$ Department of Cardiovascular Pathology, Medical-diagnostic Science and Special Therapies, University of Padua, Padua, Italy ${ }^{6}$ Clinical Pharmacology, Department of Experimental Clinical Medicine, University of Padua, Padua, Italy

${ }^{7}$ Department of Endocrinology, University of Padua, Padua, Italy

\section{Correspondence to}

Dr Alida L P Caforio, Division of Cardiology, Department of Cardiological, Thoracic and Vascular Sciences, Centro "V Gallucci",University of Padova-Policlinico, Via Giustiniani, 2, Padova 35128, Italy;

alida.caforio@unipd.it

Accepted 14 January 2010

\title{
Anti-heart and anti-intercalated disk autoantibodies: evidence for autoimmunity in idiopathic recurrent acute pericarditis
}

\author{
A L P Caforio, ${ }^{1}$ A Brucato, ${ }^{2}$ A Doria, ${ }^{3}$ G Brambilla, ${ }^{4}$ A Angelini, ${ }^{5}$ A Ghirardello, ${ }^{3}$ \\ S Bottaro, ${ }^{6} \mathrm{~F}$ Tona, ${ }^{1} \mathrm{C}$ Betterle, ${ }^{7} \mathrm{~L}$ Daliento, ${ }^{1} \mathrm{G}$ Thiene, ${ }^{5} \mathrm{~S}$ lliceto ${ }^{1}$
}

\section{ABSTRACT}

Background Idiopathic recurrent acute pericarditis (IRAP) is a rare disease of suspected, yet unproved, immune-mediated origin. The finding of serum heartspecific autoantibodies in IRAP would strengthen the autoimmune hypothesis and provide aetiology-specific non-invasive biomarkers.

Objective To assess frequency of serum anti-heart (AHA), anti-intercalated-disk (AIDA) and non-cardiacspecific autoantibodies and their clinical and instrumental correlates in patients with IRAP.

Patients 40 consecutive patients with IRAP, 25 male, aged $37 \pm 16$ years, representing a large single-centre cohort collected at a referral centre over a long time period (median 5 years, range 1-22 years). Control groups included patients with non-inflammatory cardiac disease (NICD) ( $n=160)$, ischaemic heart failure $(n=141)$ and normal subjects $(n=270)$.

Methods AHA (organ-specific, cross-reactive 1 and 2 types) and AIDA were detected in serum samples from patients, at last follow-up, and control subjects by indirect immunofluorescence (IIF) on human myocardium and skeletal muscle. Non-cardiac-specific autoantibodies were detected by IIF, and anti-Ro/SSA, anti-La/SSB by ELISA.

Results The frequencies of cross-reactive $1 \mathrm{AHA}$ and of AIDA were higher $(50 \%$; $25 \%)$ in IRAP than in NICD $(4 \%$; $4 \%)$, ischaemic (1\%; $2 \%)$ or normal subjects $(3 \% ; 0 \%)$ $(p=0.0001)$. AHA and/or AIDA were found in $67.5 \%$ patients with IRAP. Of the non-cardiac-specific antibodies, only antinuclear autoantibodies at titre $\geq 1$ / 160 were more common in IRAP (5\%) versus normal $(0.5 \%, p<0.04)$. AIDA in IRAP were associated with a higher number of recurrences $(p=0.01)$ and hospitalisations $(p=0.0001)$, high titre $(1 / 80$ or higher) AHA with a higher number of recurrences $(p=0.02)$.

Conclusions The detection of AHA and of AIDA supports the involvement of autoimmunity in the majority of patients with IRAP.

\section{INTRODUCTION}

Pericarditis may account for about $5 \%$ of presentations to emergency departments for nonischaemic chest pain. ${ }^{1}$ Recurrences occur in up to $15-32 \%$ of patients. ${ }^{2-7}$ Recurrent acute pericarditis is generally idiopathic or post-cardiac injury, and is often a frustrating problem, for both patients and doctors. ${ }^{2-7}$ Idiopathic recurrent acute pericarditis (IRAP) is a disease of suspected, yet unproved, immune-mediated pathogenesis. ${ }^{8-11}$

Patients with autoimmune conditions have a high frequency of circulating autoantibodies to the diseased target organ as well as other unaffected tissues. ${ }^{12}$ Serum anti-heart (AHA) and anti-intercalated disk autoantibodies (AIDA) detected by indirect immunofluorescence (IIF) represent autoimmune markers in patients with biopsy-proven myocarditis, dilated cardiomyopathy and in their relatives. ${ }^{13-18}$ We assessed the frequency of AHA, AIDA and of other non-cardiac-specific autoantibodies in a consecutive Italian series of patients with IRAP, as potential autoimmune markers.

\section{PATIENTS AND METHODS \\ Patients and inclusion criteria}

Sixty-three consecutive patients with recurrent acute pericarditis who fulfilled the inclusion criteria were assessed. Patients were included if they presented with a first attack of acute pericarditis defined by all the following features: typical chest pain (with or without a pericardial friction rub), suggestive electrocardiographic (ECG) changes, pericardial effusion, normal creatine kinase- $\mathrm{MB}$ and increased C-reactive protein. ${ }^{10} 19$ The minimum criteria for diagnosis of recurrences were the combination of typical chest pain, increased C-reactive protein, ECG and/or echocardiographic alterations. C-reactive protein had to be elevated in all cases during an acute attack. ${ }^{10} 19$ Only two patients were lost to follow-up; 13 patients had a post-cardiac injury syndrome; in five patients, initially labelled as 'idiopathic', a diagnosis of rheumatoid arthritis in one case and of Sjögren syndrome in four patients was made. In 43 patients the pericarditis remained 'idiopathic' at the end of a very long follow-up (mean 8.3 years, range 2-43 years); serum at last follow-up was available in 40 of them for autoantibody testing; these 40 patients are included in this study ( 25 men, aged at onset $37 \pm 16$, range $13-67$ years).

We used a previously detailed multidrug treatment protocol: non-steroidal anti-inflammatory drugs at high dosages (indometacin 75-150 mg, or aspirin 1500-2400 mg, or ibuprofen 1200-1800 mg/day); a very slow tapering of the corticosteroid if a steroid had already been started; colchicine $(0.5-1 \mathrm{mg} /$ day $)$ if tolerated; immunosuppressive agents in selected cases. ${ }^{10} 1920$

\section{AHA and AIDA testing by standard indirect immunofluorescence}

Serum samples from patients with pericarditis, taken at the last follow-up evaluation, were tested for AHA and AIDA by IIF at $1 / 10$ dilution on $4 \mu \mathrm{m}$ thick unfixed fresh frozen cryostat sections of blood 
group $\bigcirc$ normal human atrium and skeletal muscle; AHA- and AIDA-positive sera were subsequently titrated to end point by double dilutions. ${ }^{13-17}$ Organ-specific and cross-reactive 1 AHA patterns were classified as described. ${ }^{13-17}$

Briefly, organ-specific AHA gave diffuse cytoplasmic staining of atrial myocytes, but were negative on skeletal muscle; crossreactive 1 or partially organ-specific AHA gave a fine striational staining on atrium, but were negative or only weakly stained skeletal muscle; cross-reactive 2 AHA gave a broad striational pattern on longitudinal sections of heart and skeletal muscle. ${ }^{13-17}$ Absorption studies with relevant tissues had confirmed the organspecificity and cross-reactivity of the AHA patterns. ${ }^{13}$

AIDA gave a linear staining of the intercalated disks between cardiac myocytes (figure 1). Two serum samples were used as standard positive and negative controls and titrated in every assay. All sera were read blindly against these standards using a fluorescence microscope (Zeiss Axioplan 2 imaging; Zeiss, New York, USA). An additional positive control serum was titrated to assess reproducibility. Endpoint titres for this serum were reproducible within one double dilution in all assays. ${ }^{14-17}$ The frequency of AHA and of AIDA in pericarditis was compared with that observed in our established control groups of subjects with non-inflammatory cardiac disease $(n=160,80$ male, aged
$37 \pm 17$ years, of whom $n=55$ had rheumatic heart disease, $n=67$ hypertrophic cardiomyopathy, $\mathrm{n}=38$ congenital defects), ischaemic heart disease ( $n=141,131$ male, aged $44 \pm 14$ years) and normal subjects $(n=270,123$ male, age $35 \pm 11) .{ }^{14-17}$ Fortyone of the 141 ischaemic patients, aged $47 \pm 12$ years, 28 male; 31 in New York Heart Association (NYHA) III and 10 in NYHA IV, had had a myocardial infarct 6 months to 10 years (median 2 years) previously; ejection fraction ranged from 16\% to $44 \%$

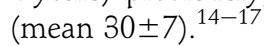

\section{Non-cardiac-specific autoantibody tests}

Antinuclear antibodies were tested by IIF on HEp2 cells and anti-mitochondrial antibodies by IIF on rat liver and kidney. Other non-cardiac-specific autoantibodies, including antithyroid, anti-gastric parietal cell, anti-islet cell, and anti-adrenal cell were tested by IIF on rat thyroid gland, rat stomach, human pancreas, human adrenal gland, respectively. For all these antibodies, 375 healthy subjects, 211 male and 164 female, aged $38 \pm 12$ years were considered as controls.

Anti-Ro/SSA (52 kDa and $60 \mathrm{kDa}$ ) and anti-La/SSB antibodies were tested using a commercially available ELISA (INOVA Diagnostics, San Diego, California, USA); controls included 75 subjects, 46 male, aged $37 \pm 3$.
Figure 1 Anti-heart autoantibody (AHA) immunofluorescence patterns. Blood group 0 normal human atrium (left panels) and skeletal muscle (right panels) stained with $(A, B)$ AHAnegative serum from a normal subject. No myocyte or muscle staining is present. $(C, D)$ Serum from a patient with idiopathic recurrent acute pericarditis (IRAP), containing crossreactive (partially organ-specific) AHA. A fine striational fluorescence is visible on atrial tissue; skeletal muscle is weakly positive. (E,F) Serum from a patient with IRAP, containing both organ-specific AHA and antiintercalated-disk autoantibodies (AIDA). Organ-specific AHA give a diffuse cytoplasmic indirect

immunofluorescence technique staining on atrial myocytes, AIDA produce a linear staining of the intercalated disks between cardiac myocytes; skeletal muscle is negative. Magnification $\times 400$.
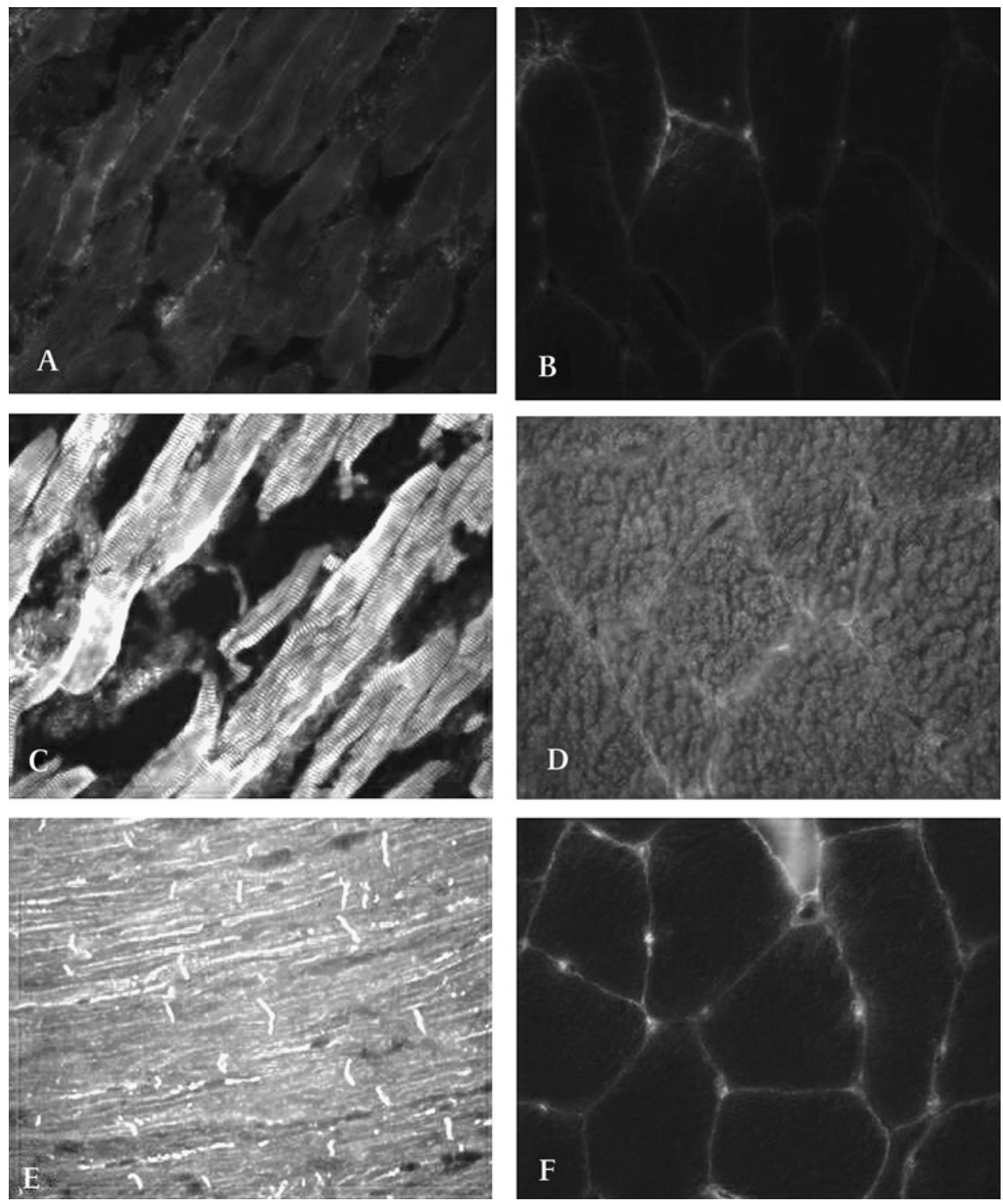


\section{Statistical analysis}

Results for quantitative features are given as means \pm SD. Student $t$ test, one-way analysis of variance, $\chi^{2}$ (with Yates's correction) or Fisher's exact test was used as appropriate. All $p$ values were two tailed; $p$ values $<0.05$ were considered to indicate statistical significance. All antibodies were tested in the same sample (eg, at the same time point). All statistical analyses were performed using the StatView software version 5.0 for Apple Macintosh (SAS Institute).

\section{RESULTS}

\section{Clinical and diagnostic features in patients with IRAP}

Main clinical and diagnostic features of the patients with IRAP are detailed in table 1. At the time of last follow-up, when serum was taken for the immunological antibody tests, 21 (52\%) were still receiving treatment as detailed in table 1 , all but one were in NYHA class I, one was mildly symptomatic (class II), with a normal echocardiographic left ventricular ejection fraction.

\section{Frequency of AHA, AIDA and other autoantibodies in IRAP}

The frequency and AHA of IgG class in IRAP and controls is shown in table 2. Representative examples of AHA and AIDA patterns are shown in figure 1 . The frequencies of cross-reactive 1 AHA and AIDA were higher in IRAP than in control subjects $(p=0.0001)$. Conversely, the frequencies of cross-reactive 2 and organ-specific AHA were similar in IRAP, and control subjects respectively $(p=N S)$. AHA of any type and/or AIDA were found in $27(67.5 \%)$ patients with pericarditis; AHA of the crossreactive 1 type and/or AIDA were found in 22 (55\%). AHA titres among the 25 antibody positive sera were as follows: $1 / 10$ in eight $(32 \%), 1 / 40$ in $10(40 \%), \geq 1 / 80$ in seven (28\%). AIDA titres among the 10 antibody-positive sera were as follows: $1 / 10$ in six $(60 \%), 1 / 40$ in three $(30 \%)$ and $1 / 80$ in 1 (10\%).

The frequency of other autoantibodies in the study groups is shown in table 3 . The frequency of positive (titre $\geq 1 / 160$ ) antinuclear antibody was higher in pericarditis than in normal subjects $(p<0.04)$. Other non-cardiac-specific antibodies were all

Table 1 Clinical features in patients with idiopathic recurrent acute pericarditis $(n=40)$

\begin{tabular}{|c|c|}
\hline \multicolumn{2}{|l|}{ Clinical course: } \\
\hline Symptom duration (years, median) & 5, range $1-22$ \\
\hline Relapses/year & $1.8 \pm 1$, range $1-5$ \\
\hline Hospital admissions & $3 \pm 2$, range $1-9$ \\
\hline Follow-up (years), median & 8 , range $1-43$ \\
\hline $\begin{array}{l}\text { New York Heart Association at last } \\
\text { follow-up, } n(\%)\end{array}$ & I, 39 (98\%); II, 1 (2\%) \\
\hline \multicolumn{2}{|c|}{ Two-dimensional echocardiography (last follow-up): } \\
\hline Mean end-diastolic diameter (mm) & $48 \pm 3$ \\
\hline Mean end-diastolic septal thickness (mm) & $10 \pm 1$ \\
\hline Mean left ventricular ejection fraction (\%) & $62 \pm 4$ \\
\hline Treatment at any time of follow-up, $n,(\%)$ : & $40(100)$ \\
\hline Steroids & $34(85)$ \\
\hline Colchicine & $34(85)$ \\
\hline Non-steroidal anti-inflammatory drugs & $40(100)$ \\
\hline $\begin{array}{l}\text { Immunosuppressant agents* } \\
\text { pericardiocentesis and/or pericardiectomy }\end{array}$ & $7(17)$ \\
\hline Treatment at last follow-up, $\mathrm{n}(\%)$ : & $21(52)$ \\
\hline Steroids & $16(40)$ \\
\hline Colchicine & $17(42)$ \\
\hline Non-steroidal anti-inflammatory drugs & $13(32)$ \\
\hline Any drug combination & $16(40)$ \\
\hline
\end{tabular}

Table 2 Frequency of anti-heart autoantibodies (AHA) and of antiintercalated-disk autoantibodies (AIDA) by immunofluorescence in patients with idiopathic recurrent acute pericarditis (IRAP) and in controls

\begin{tabular}{lllll}
\hline & $\begin{array}{l}\text { Organ-specific } \\
\text { AHA } \\
\mathbf{n}(\%)\end{array}$ & $\begin{array}{l}\text { Cross-reactive 1 } \\
\text { AHA } \\
\mathbf{n}(\%)\end{array}$ & $\begin{array}{l}\text { Cross-reactive } \\
\text { AHA } \\
\mathbf{n}(\%)\end{array}$ & $\begin{array}{l}\text { AIDA } \\
\mathbf{n}(\%)\end{array}$ \\
\hline $\begin{array}{l}\text { IRAP }(\mathrm{n}=40) \\
\text { Non-inflammatory }\end{array}$ & $\begin{array}{l}2(5) \\
\text { cardiac disease } \\
\text { (n=160) }\end{array}$ & $\begin{array}{c}20(50)^{*},+ \\
6(4)\end{array}$ & $\begin{array}{l}3(7.5) \\
5(3)\end{array}$ & $\begin{array}{l}10(25)^{*}, \dagger \\
6(4)\end{array}$ \\
$\begin{array}{l}\text { Ischaemic heart } \\
\text { failure ( } \mathrm{n}=141)\end{array}$ & $1(1)$ & $1(1)$ & $8(6)$ & $3(2)$ \\
$\begin{array}{l}\text { Normal subjects } \\
\text { (n=270) }\end{array}$ & $7(2.5)$ & $8(3)$ & $8(3)$ & $0(0)$ \\
\hline
\end{tabular}

${ }^{*} \mathrm{p}=0.0001$ versus non-inflammatory heart disease or ischaemic heart failure $t p=0.0001$ versus normal subjects.

negative in IRAP, but two were parietal cell antibody positive and one anti-Ro/SSA positive. The patient who was anti-Ro/SSA positive did not have Sjögren syndrome. None of the patients with IRAP with non-cardiac-specific antibodies had co-occurring autoimmune conditions. Overall $27(67.5 \%)$ patients with IRAP were positive for AHA, AIDA and/or other autoantibodies.

\section{Clinical correlates of AHA, AIDA and other autoantibodies in IRAP}

Comparison of clinical and diagnostic features in relation to AHA and AIDA status is shown in tables 4-6. There were no differences between patients with AHA and those without (table 4). AHA-positive patients tended to be more frequently receiving combined drug treatment at last follow-up compared with those without $(p=0.07)$. Conversely, patients with high titre $(\geq 1 / 80)$ AHA had longer symptom duration $(p=0.006)$, higher number of recurrences $(p=0.02)$, higher end-diastolic posterior wall thickness $(p=0.02)$ and tended to have longer follow-up $(p=0.06)$ and more frequent treatment with combined treatment at the last follow-up $(p=0.08)$ compared with those with low-titre $(<1 / 80)$ or negative AHA (table 5). AIDA-positive patients had a higher number of recurrences $(p=0.01)$ and of hospitalisations $(p=0.0001)$; AIDA-positive status tended to be more common among patients with refractory symptoms, treated at some stage during follow-up with immunosuppressive drugs and/or pericardiocentesis or pericardiectomy $(p=0.05)$ (table 6). Sensitivities, specificities, positive and negative predictive values for the various AHA and AIDA patterns in IRAP are shown in table 7.

\section{DISCUSSION}

In this study we found an increased frequency of cross-reactive 1 AHA and AIDA in patients with IRAP compared with control

Table 3 Frequency of non-cardiac-specific autoantibodies by immunofluorescence and ELISA in patients with idiopathic recurrent acute pericarditis (IRAP) and in normal control subjects

\begin{tabular}{llll}
\hline & IRAP $(\mathbf{n = 4 0 )}$ & Normal subjects $(\mathbf{n}=\mathbf{3 7 5})$ & $\mathbf{p}$ Value \\
\hline Anti-mitochondrial, $\mathrm{n}(\%)$ & $0(0)$ & $1(0.3)$ & NS \\
Antinuclear, titre $\geq 1 / 160$ & $2(5)$ & $2(0.5)$ & 0.04 \\
Anti-thyroid, $\mathrm{n}(\%)$ & $0(0)$ & $26(7)$ & $\mathrm{NS}$ \\
Anti-islet cell, $\mathrm{n}(\%)$ & $0(0)$ & $4(1)$ & $\mathrm{NS}$ \\
Anti-parietal cell, $\mathrm{n}(\%)$ & $2(5)$ & $7(1.8)$ & $\mathrm{NS}$ \\
Anti-adrenal cell, $\mathrm{n}(\%)$ & $0(0)$ & $0(0)$ & $\mathrm{NS}$ \\
& $(\mathrm{n}=40)$ & $(\mathrm{n}=75)$ & \\
Anti-Ro/SSA & $1(2)$ & $1(1)$ & $\mathrm{NS}$ \\
Anti-La/SSB & $0(0)$ & $0(0)$ & $\mathrm{NS}$ \\
\hline
\end{tabular}


Table 4 Clinical features and anti-heart autoantibody (AHA) status in idiopathic recurrent acute pericarditis

\begin{tabular}{lccc}
\hline & $\begin{array}{c}\text { AHA positive } \\
(\mathbf{n = 2 5})\end{array}$ & $\begin{array}{c}\text { AHA negative } \\
(\mathbf{n = 1 5 )}\end{array}$ & p Value \\
\hline Age at onset (years) & $38 \pm 16$ & $36 \pm 16$ & NS \\
Male/female & $15 / 10$ & $10 / 5$ & NS \\
Symptoms (years) & $6 \pm 9$ & $4 \pm 3$ & NS \\
Follow-up (years) & $9 \pm 11$ & $7 \pm 4$ & NS \\
Recurrences & $7 \pm 9$ & $5 \pm 3$ & NS \\
Recurrences/year & $2 \pm 1$ & $1 \pm 1$ & NS \\
Hospital admissions & $3 \pm 2$ & $2.5 \pm 1$ & NS \\
End-diastolic septal thickness (mm) & $10 \pm 1$ & $9.5 \pm 1$ & NS \\
End-diastolic posterior wall thickness & $9 \pm 2$ & $8 \pm 1$ & NS \\
(mm) & & & \\
Left ventricular ejection fraction (\%) & $61 \pm 4$ & $64 \pm 3$ & NS \\
Immunosuppressant agents and/or & $6(24)$ & $1(7)$ & NS \\
pericardiectomy (\%) & & & \\
Drug combination at last follow-up (\%) & $10(40)$ & $2(13)$ & 0.07 \\
\hline
\end{tabular}

subjects, in keeping with autoimmune involvement in at least $55 \%$ of patients. Cross-reactive $1 \mathrm{AHA}$, which are increased in IRAP, have partial cardiac specificity and are also more frequently found in myocarditis or dilated cardiomyopathy than in controls. ${ }^{13-18}$ Since true IRAP is a rare disease, the series reported here is the largest single-centre cohort collected at a referral centre during a long period and the first to undergo immunological screening for cardiac autoimmunity. Other previously recognised autoimmune features in IRAP ${ }^{12}$ include its chronic/recurrent course; detection of proinflammatory cytokines in the pericardial fluid but not in plasma, suggesting a local inflammatory reaction ${ }^{8}$; familial aggregation and a weak association with the HLA-B14 and DRB1*01 alleles that have been associated with other immune-mediated conditions ${ }^{9}{ }^{21}$; and efficacy of anti-inflammatory or immunosuppressive treatments. $^{9-11} 20-23$

In this study positive AIDA status was associated with a high number of relapses, hospitalisations and refractory symptoms, suggesting higher immune activation during the "hot" phases of pericardial inflammation. In addition, higher AHA titres were associated with longer symptom duration and high number of recurrences. These findings further reinforce the autoimmune

Table 5 Clinical features and anti-heart autoantibody (AHA) titre in idiopathic recurrent acute pericarditis

\begin{tabular}{|c|c|c|c|}
\hline & $\begin{array}{l}\text { High titre }(\geq 1 / 80) \\
\text { AHA }(n=7)\end{array}$ & $\begin{array}{l}\text { Low titre }(<1 / 80) \text { or } \\
\text { negative AHA }(n=33)\end{array}$ & p Value \\
\hline Age at onset (years) & $44 \pm 21$ & $36 \pm 14$ & NS \\
\hline Male/female & $4 / 3$ & $21 / 12$ & NS \\
\hline Symptoms (years) & $12 \pm 15$ & $4 \pm 3$ & 0.006 \\
\hline Follow-up (years) & $14 \pm 16$ & $7 \pm 6$ & 0.06 \\
\hline Recurrences & $12 \pm 16$ & $5 \pm 3$ & 0.02 \\
\hline Recurrences/year & $2 \pm 1$ & $2 \pm 1$ & NS \\
\hline Hospital admissions & $3 \pm 2$ & $3 \pm 2$ & NS \\
\hline $\begin{array}{l}\text { End-diastolic septal } \\
\text { thickness (mm) }\end{array}$ & $10 \pm 1$ & $9 \pm 1$ & NS \\
\hline $\begin{array}{l}\text { End-diastolic posterior wall } \\
\text { thickness }(\mathrm{mm})\end{array}$ & $10 \pm 2$ & $8 \pm 1$ & 0.02 \\
\hline $\begin{array}{l}\text { Left ventricular ejection } \\
\text { fraction }(\%)\end{array}$ & $61 \pm 4$ & $62 \pm 4$ & NS \\
\hline $\begin{array}{l}\text { Immunosuppressant agents } \\
\text { and/or pericardiectomy(\%) }\end{array}$ & $5(71)$ & $15(45)$ & NS \\
\hline $\begin{array}{l}\text { Drug combination at last } \\
\text { follow-up (\%) }\end{array}$ & $4(57)$ & $8(24)$ & 0.08 \\
\hline
\end{tabular}

Table 6 Clinical features and anti-intercalated-disk autoantibody (AIDA) status in idiopathic recurrent acute pericarditis

\begin{tabular}{lccc}
\hline & $\begin{array}{l}\text { AIDA positive } \\
(\mathbf{n = 1 0 )}\end{array}$ & $\begin{array}{l}\text { AIDA negative } \\
(\mathbf{n = 3 0 )}\end{array}$ & p Value \\
\hline Age at onset (years) & $36 \pm 16$ & $38 \pm 16$ & NS \\
Male/female & $6 / 4$ & $19 / 11$ & NS \\
0-S positive & $1(10)$ & $1(3)$ & NS \\
Symptoms (years) & $6 \pm 7$ & $5 \pm 8$ & NS \\
Follow-up (years) & $12 \pm 9$ & $7 \pm 8$ & NS \\
Recurrences & $11 \pm 14$ & $5 \pm 3$ & 0.01 \\
Recurrences/year & $2 \pm 1$ & $2 \pm 1$ & NS \\
Hospital admissions & $5 \pm 3$ & $2 \pm 1$ & 0.0001 \\
End-diastolic septal thickness (mm) & $9.5 \pm 1$ & $10 \pm 1$ & NS \\
End-diastolic posterior wall thickness & $8 \pm 1$ & $9 \pm 1$ & NS \\
(mm) & & & \\
Left ventricular ejection fraction (\%) & $62 \pm 3$ & $62 \pm 4$ & NS \\
Immunosuppressant agents and/or & $4(40)$ & $3(10)$ & 0.05 \\
pericardiectomy, $n$ (\%) & & & \\
Drug combination at last follow-up, $\mathrm{n}(\%)$ & $2(20)$ & $10(33)$ & NS \\
\hline
\end{tabular}

hypothesis in IRAP. Cross-reactive 1 AHA and AIDA frequency was high in spite of a long duration of symptoms and in some instances of immunosuppressive treatment. However, most immunosuppressive drugs do not lower autoantibody titres. ${ }^{24}$ In keeping with this, we have recently reported that positive AHA status is an independent predictor of high rejection burden in patients with a longstanding heart transplant receiving chronic dual or triple immunosuppressive regimens comparable to those used in IRAP. ${ }^{25}$

Surprisingly, the frequency of organ-specific AHA was similar in patients with IRAP and in controls, whereas in biopsy-proven myocarditis and in dilated cardiomyopathy, organ-specific AHA frequency is increased compared with control groups. ${ }^{13-18}$ There are possible explanations for the discrepancy. First, in autoimmune disease, different antibody specificities are associated with distinct clinical phenotypes ${ }^{12}$; thus cross-reactive 1 AHA may be more prevalent in pericarditis, organ-specific AHA in myocarditis. This view is supported by earlier reports of serum cross-reactive AHA in other forms of suspected immune-mediated pericarditis-for example, the post-pericardiotomy syndrome or the Dressler syndrome. ${ }^{26} 27$ Second, in autoimmune disease some autoantibodies are detected earlier in the disease course and others in advanced phases and/or during relapses. ${ }^{28}$ Longitudinal studies in IRAP should clarify the temporal sequence of appearance of each autoantibody specificity; it may be that organ-specific AHA are early markers, followed by AIDA and cross-reactive AHA in advanced IRAP. This would mirror the temporal sequence observed in patients with dilated cardiomyopathy, where organ-specific AHA are early markers that become undetectable in advanced disease. ${ }^{13-15} 29$

Three of the autoantigens recognised by the AHA detected by IIF in dilated cardiomyopathy were identified by us as $\alpha$ and $\beta$ myosin heavy chain and myosin light chain- $1 \mathrm{v}$ isoforms by western blotting ${ }^{30}$ and confirmed by others. ${ }^{31} 32$ It is highly likely that the same autoantigens are also responsible for AHA and AIDA found in IRAP, since AHA and AIDA in IRAP (figure 1) and in biopsy-proven myocarditis with or without concurrent pericarditis ${ }^{16}$ or in dilated cardiomyopathy sera ${ }^{13} 1417$ exhibit the same localisation patterns by IIF. The autoantigen(s) responsible for AIDA have not yet been identified, but there are many candidates. ${ }^{33}$ So far this is the first study showing increased AHA and AIDA in IRAP. Small variable amounts of sera were 
Table 7 Sensitivities, specificities, positive (PPV) and negative predictive values (NPV) of anti-heart autoantibody (AHA) and anti-intercalated-disk AIDA autoantibody patterns in idiopathic recurrent acute pericarditis (IRAP)

\begin{tabular}{|c|c|c|c|c|c|c|c|}
\hline & IRAP & Normal subjects & p Value & Sensitivity (\%) & Specificity (\%) & PPV (\%) & NPV (\%) \\
\hline \multicolumn{8}{|l|}{ AHA (any pattern): } \\
\hline Positive/negative & $25 / 15$ & $23 / 247$ & 0.0001 & 62 & 91 & 52 & 94 \\
\hline \multicolumn{8}{|c|}{ AHA (organ-specific or cross-reactive 1): } \\
\hline Positive/negative & $22 / 18$ & $15 / 255$ & 0.0001 & 55 & 94 & 59 & 93 \\
\hline \multicolumn{8}{|l|}{ AIDA: } \\
\hline Positive/negative & $10 / 30$ & $0 / 270$ & 0.0001 & 25 & 100 & 100 & 90 \\
\hline \multicolumn{8}{|c|}{ AHA (any pattern) and/or AIDA positive: } \\
\hline Positive/negative & $27 / 13$ & $23 / 247$ & 0.0001 & 54 & 95 & 67.5 & 91.5 \\
\hline \multicolumn{8}{|c|}{ AHA (organ-specific or cross-reactive 1) and/or AIDA: } \\
\hline Positive/negative & $22 / 18$ & $15 / 255$ & 0.0001 & 59 & 93 & 55 & 94 \\
\hline
\end{tabular}

available. This prevented us from further testing for autoantigen specificity. We aim at identifying additional autoantigens in newly diagnosed patients; in keeping with other autoimmune diseases, autoantigens involved in early disease stages are more closely related to primary pathogenetic mechanisms and are more likely to be pathogenetically relevant than those which have a role in secondary immunopathogenesis. ${ }^{12}$ The search for additional antibodies should be extended using pericardial substrate, though it is cumbersome to obtain normal pericardial tissue for analysis. For this reason it is presently unknown whether AHA and AIDA cross react with pericardial tissue.

So far, evidence for a direct pathogenic role of the AHA and AIDA detected by IIF in patients with myocarditis or dilated cardiomyopathy is lacking. ${ }^{13-17} 34$ However, animal data as well as in vitro and early clinical observations suggest that some of the antibodies (eg, anti- $\beta 1$ adrenergic receptors, anti-myosin, anti-troponin) detected by other immunological techniques may have a functional role in a patient subset. ${ }^{34-46}$ Further work is needed to clarify this issue in IRAP, although the lack of clinical and echocardiographic evidence of ventricular dysfunction in our patients at the time of serum testing argues against a direct pathogenic role of AHA or AIDA.

\section{Study limitations}

It is not known if myocarditis was associated with pericarditis in our patients. None of the patients had echocardiographic findings suggestive of myocardial involvement (regional or global wall motion abnormalities, increased wall thickness) or increased creatine kinase- $\mathrm{MB},{ }^{47}$ but endomyocardial biopsy was not performed. Since the diagnosis of myocarditis is based on endomyocardial biopsy, myocarditis cannot be theoretically excluded. $^{48} 49$ However, typical acute pericarditis findings as those described in this study for the first episode were uncommon in our recent large prospective series of biopsyproven myocarditis. ${ }^{16}$ In addition, biopsy-proven myocarditis leads to death or heart transplantation in $27 \%$ of patients at 6 years ${ }^{16}$; conversely, IRAP has a benign prognosis, as confirmed in the present cohort with a long follow-up. ${ }^{19}$ In support of our conservative approach, none of the patients with IRAP described here presented with one of the clinical scenarios (eg, heart failure or life-threatening arrhythmia) that warrant endomyocardial biopsy according to the recent guidelines. ${ }^{50}$ Our hypothesis, in keeping with previous observations, ${ }^{57}$ is that myocarditis is unlikely to be present in IRAP.
Current guidelines suggest that the diagnosis of autoimmune pericarditis should be based upon pericardial/epicardial biopsy findings. ${ }^{6}$ A limitation of our study is that pericardial biopsy was not performed. However, experts advocate that once collagen vascular disease, previous cardiac surgery or myocardial infarction are ruled out, as for our patients, relapsing pericarditis is almost invariably immune-mediated with or without a viral trigger. ${ }^{47}$ Pericardial biopsy is not widely used. ${ }^{47}$ Last but not least IRAP has a benign prognosis, as reported ${ }^{1} 19 \quad 45$ and confirmed here, thus an invasive approach may not be justified, at least in the majority of patients. ${ }^{4} 7$

\section{CONCLUSION}

The high prevalence of AHA and AIDA shown here strongly suggests that IRAP is an autoimmune disease in a large subset of patients. Whether or not these autoantibodies may provide clinically useful biomarkers of pericarditis relapses and/or noninvasive tools to tailor immunosuppressive treatment warrants future assessment.

Funding This work was supported by the Ministry for University Scientific and Technological Research (MURST) Target Projects (1999-2000, Myocarditis: therapeutic impact of etiological diagnosis based upon molecular and immunological findings; 2003-2005, Myocarditis: identification of clinical, molecular and immunological markers for risk stratification), and the Ministry of Health Target Project (2004-2007, Inflammatory cardiomyopathy), Rome, Italy.

\section{Competing interests None.}

Ethics approval This study was conducted with the approval of the Ospedale Niguarda, Milan, Italy.

Patient consent Obtained.

Provenance and peer review Not commissioned; externally peer reviewed.

\section{REFERENCES}

1. Launbjerg J, Fruergaard $\mathrm{P}$, Hesse $\mathrm{B}$, et al. Long-term risk of death, cardiac events and recurrent chest pain in patients with acute chest pain of different origin. Cardiology 1996;87:235-40

2. Imazio M, Bobbio M, Cecchi $\mathrm{E}$, et al. Colchicine as first-choice therapy for recurrent pericarditis: results of the CORE (COlchicine for REcurrent pericarditis) trial. Arch Int Med 2005;165:1987-91.

3. Spodick DH. Acute pericarditis: current concepts and practice. JAMA 2003;289:1150-3.

4. Shabetai R. Recurrent pericarditis. Recent advances and remaining questions. Circulation 2005:112:1921-3.

5. Soler-Soler J, Sagrista-Sauleda J, Permanyer-Miralda G. Relapsing pericarditis. Heart 2004;90:1364-8.

6. Maisch B, Seferovic PM, Ristic AD, et al. Guidelines on the diagnosis and management of pericardial diseases executive summary; The Task force on the 
diagnosis and management of pericardial diseases of the European society of cardiology. Eur Heart J 2004;25:587-610.

7. Fowler NO. Recurrent pericarditis. Cardiol Clin 1990;8:621-6.

8. Pankuweit $\mathbf{S}$, Wadlich $\mathrm{A}$, Meyer $\mathrm{E}$, et al. Cytokine activation in pericardial fluids in different forms of pericarditis. Herz 2000;25:748-54.

9. Brucato A, Brambilla G. Recurrent idiopathic pericarditis: familial occurrence. Int J Cardiol 2005;102:529 (letter).

10. Brucato A, Brambilla G, Adler $Y$, et al. Therapy of recurrent acute pericarditis: a rheumatological solution? Clin Exp Rheumatol 2006:24:45-50.

11. Marcolongo R, Russo R, Laveder F, et al. Immunosuppressive therapy prevents recurrent pericarditis. J Am Coll Cardiol 1995;26:1276-9.

12. Rose NR, Bona C. Defining criteria for autoimmune diseases (Witebsky's postulates revisited). Immunol Today 1993;14:426-8.

13. Caforio ALP, Bonifacio E, Stewart JT, et al. Novel organ-specific cardiac autoantibodies in dilated cardiomyopathy. J Am Coll Cardiol 1990;15:1527-34.

14. Caforio ALP, Keeling PJ, Zachara E, et al. Evidence from family studies for autoimmunity in dilated cardiomyopathy. Lancet 1994;344:773-7.

15. Caforio ALP, Mahon NG, Baig KM, et al. Prospective familial assessment in dilated cardiomyopathy: cardiac autoantibodies predict disease development in asymptomatic relatives. Circulation 2007:115:76-83.

16. Caforio ALP, Calabrese F, Angelini A, et al. A prospective study of biopsy-proven myocarditis: prognostic relevance of clinical and etiopathogenetic features at diagnosis. Eur Heart J 2007;28:1326-33.

17. Caforio ALP, Haven AJ, Goldman JH, et al. Frequency and clinical correlates of antiintercalated disk autoantibodies in dilated cardiomyopathy [abstract]. J Am Coll Cardiol 1996:27 (Suppl A):P227.

18. Maisch B, Deeg P, Liebau G, et al. Diagnostic relevance of humoral and cytotoxic immune reactions in primary and secondary dilated cardiomyopathy. Am J Cardiol 1983:52:1072-8.

19. Brucato A, Brambilla G, Moreo A, et al. Long-term outcomes in difficult-to-treat patients with recurrent pericarditis. Am J Cardiol 2006:98:267-71.

20. Brucato A, Brambilla G, Adler $Y$, et al. Colchicine for recurrent acute pericarditis. Arch Intern Med 2006;166:696 (letter)

21. Brucato A, Shinar Y, Brambilla G, et al. Idiopathic recurrent acute pericarditis:familial fever mutations and disease evolution in a large cohort of Caucasian patients. Lupus 2005; 14:670-4.

22. Imazio $\mathbf{M}$, Bobbio $\mathrm{M}$, Cecchi $\mathrm{E}$, et al. Colchicine in addition to conventional therapy for acute pericarditis. Circulation 2005;112:2012-16.

23. Artom G, Koren-Morag N, Brucato A, et al. Pretreatment with corticosteroids attenuates the efficacy of colchicine in preventing recurrent pericarditis: a multicenter all-case analysis. Eur Heart J 2005;26:723-7.

24. MacGregor AJ, Silman AJ. Rheumatoid arthritis: classification and epidemiology. In: Klippel JH, Dieppe PA, eds. Rheumatology. 2nd edn. London: Mosby, 1998:5.2.2-6

25. Caforio ALP, Vinci A, Angelini A, et al. Circulating anti-heart autoantibodies are noninvasive markers of high cellular rejection burden in heart transplantation [abstract]. J Heart Lung Transplant 2009:28(2S):S86-7.

26. Maisch B, Berg PA, Kocksiek K. Clinical significance of immunopathological findings in patients with post-pericardiotomy sindrome. I relevance of antibody pattern. Clin Exp Immunol 1979;38:189-97.

27. Kaplan MH, Frengley JD. Autoimmunity to the heart in cardiac disease. Current concepts of the relation of autoimmunity to rheumatic fever, postcardiotomy and postinfarction syndromes and cardiomyopathies. Am J Cardiol 1969:24:459-73.

28. Arbuckle MR, McClain MT, Rubertone MV, et al. Development of autoantibodies before the clinical onset of systemic lupus erythematosus. $N$ Engl J Med 2003:349:1526-33.

29. Caforio ALP, Goldman JH, Baig KM, et al. Cardiac autoantibodies in dilated cardiomyopathy become undetectable with disease progression. Heart 1997;77:62-7.
30. Caforio ALP, Grazzini M, Mann JM, et al. Identification of the $\alpha$ and $\beta$ myosin heavy chain isoforms as major autoantigens in dilated cardiomyopathy. Circulation 1992;85:1734-42.

31. Latif N, Baker CS, Dunn MJ, et al. Frequency and specificity of antiheart antibodies in patients with dilated cardiomyopathy detected using SDS-PAGE and western blotting. J Am Coll Cardiol 1993;22:1378-84.

32. Lauer B, Schannwell M, Kuhl U, et al. Antimyosin autoantibodies are associated with deterioration of systolic and diastolic left ventricular function in patients with chronic myocarditis. J Am Coll Cardiol 2000;35:11-18.

33. Perriard JC, Hirschy A, Ehler E. Dilated cardiomyopathy: a disease of the intercalated disk? Trends Cardiovasc Med 2003;13:30-8.

34. Caforio ALP, Tona F, Bottaro $S$, et al. Clinical implications of anti-heart autoantibodies in myocarditis and dilated cardiomyopathy. Autoimmunity 2008;41:35-45.

35. Okazaki T, Tanaka Y, Nishio R, et al. Autoantibodies against cardiac troponin I are responsible for dilated cardiomyopathy in PD-1-deficient mice. Nat Med 2003;9:1477-83

36. Jahns R, Boivin V, Hein L, et al. Direct evidence for a beta 1-adrenergic receptordirected autoimmune attack as a cause of idiopathic dilated cardiomyopathy. J Clin Invest 2004:113:1419-29.

37. Kuan AP, Zuckier L, Liao L, et al. Immunoglobulin isotype determines pathogenicity in antibody-mediated myocarditis in naïve mice. Circ Res 2000;86:281-5.

38. Li Y, Heuser JS, Cunningham LC, et al. Mimicry and antibody-mediated cell signaling in autoimmune myocarditis. J Immunol 2006;177:8234-40.

39. Warraich RS, Griffiths E, Falconar A, et al. Human cardiac myosin autoantibodies impair myocyte contractility: a cause-and-effect relationship. FASEB 2006;20:651-60

40. Nikolaev V0, Boivin V, Stork S, et al. A novel fluorescence method for the rapid detection of functional $\beta 1$-adrenergic receptor autoantibodies in heart failure. J Am Coll Cardiol 2007:50:423-31.

41. Okazaki T, Honjo T. Pathogenic roles of cardiac autoantibodies in dilated cardiomyopathy. Trends Mol Med 2005;11:322-6.

42. Jahns R, Boivin V, Lohse MJ. $\beta 1$-adrenergic receptor function, autoimmunity and pathogenesis of dilated cardiomyopathy. Trends Cardiovasc Med 2006:16:20-4.

43. Staudt A, Eichler P, Trimpert C, et al. Fc $\gamma$ receptors lla on cardiomyocytes and their potential functional relevance in dilated cardiomyopathy. J Am Coll Cardiol 2007:49:1684-92.

44. Mobini R, Staudt A, Felix SB, et al. Hemodynamic improvement and removal of autoantibodies against the $\beta 1$-adrenergic receptor by immunoadsorption therapy in dilated cardiomyopathy. J Autoimmun 2003;20:345-50.

45. Mann DL. Autoimmunity, immunoglobulin adsorption and dilated cardiomyopathy: has the time come for randomized clinical trials? J Am Coll Cardiol 2001:38:184-6.

46. Jane-wit D, Altuntas CZ, Johnson JM, et al. $\beta 1$-adrenergic receptor autoantibodies mediate dilated cardiomyopathy by agonistically inducing cardiomyocyte apoptosis. Circulation 2007:116:399-410

47. Imazio M, Demichelis B, Cecchi $\mathrm{E}$, et al. Cardiac troponin I in acute pericarditis Cardiac troponin I in acute pericarditis. J Am Coll Cardiol 2003:42:2144-8.

48. Richardson P, McKenna WJ, Bristow M, et al. Report of the 1995 World Health Organization/International Society and Federation of Cardiology Task Force on the definition and classification of cardiomyopathies. Circulation 1996:93:841-2.

49. Dennert R, Crijns HJ, Heymans S. Acute viral myocarditis. Eur Heart $J$ 2008;29:2073-82.

50. Cooper LT, Baughman KL, Feldman AM, et al. The role of endomyocardial biopsy in the management of cardiovascular disease: a scientific statement from the American Heart Association, the American College of Cardiology, and the European Society of Cardiology. Endorsed by the Heart Failure Society of America and the Heart Failure Association of the European Society of Cardiology. Circulation 2007:116:2216-33. 